\title{
La légionellose
}

Elisabeth Bouvet

> Bien qu'on ne retrouve pas l'origine de la contamination dans la moitié des cas de légionellose humaine, on sait que cette maladie est la conséquence de la contamination presque obligatoire des réseaux d'installations d'eau chaude par Legionella pneumophila, et l'inhalation par l'homme de gouttelettes infectées. La pathologie consiste le plus souvent en une pneumopathie relativement grave. La maîtrise du niveau de contamination des différentes installations hydriques productrices d'aérosols est impérative pour éviter les conséquences sanitaires graves, qui ne peuvent être prévenues par une action sur la cible humaine. La plupart des réservoirs actuellement identifiés sont les tours aéroréfrigérantes et les réseaux de distribution d'eau chaude. La prise en compte de ce risque dans les établissements hospitaliers ou thermaux a conduit à la mise en œuvre de nombreuses mesures de désinfection et de contrôle, qui commencent à montrer une certaine efficacité sur la survenue de nouveaux cas dans ces établissements, aujourd'hui en nette diminution. <

La légionellose a été décrite pour la première fois en 1976 à Philadelphie, aux États-Unis, à l'occasion d'une épidémie de pneumopathies graves survenant chez d'anciens légionnaires américains réunis en congrès. La bactérie responsable de la maladie, découverte à cette occasion et baptisée Legionella, était d'origine hydrique, comme l'a démontré l'enquête réalisée dans l'hôtel où se déroulait le congrès; le réservoir en était le système de climatisation humide. Les malades possédaient pour la plupart des facteurs favorisants, en l'occurrence un âge supérieur à 50 ans et une maladie bronchopulmonaire chronique liée à un tabagisme ancien [1].

Ces dernières années, plusieurs épidémies occasionnant des dizaines de cas ont été décrites en Amérique du Nord et en Europe, notamment en France. Les sources d'infection identifiées sont aujourd'hui toujours les mêmes : systèmes de climatisation humide, tours aéro-

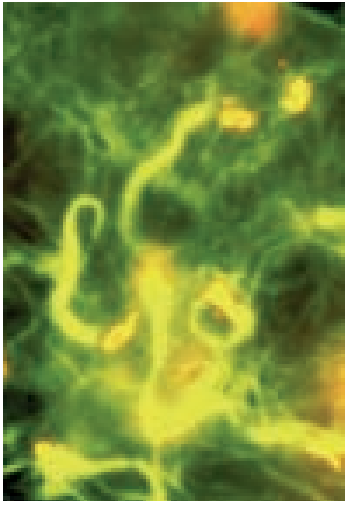

Service

des maladies infectieuses

et tropicales,

Hôpital Bichat,

46, rue Henri-Huchard,

réfrigérantes, systèmes

75018 Paris, France.

d'eau chaude sanitaire

elisabeth.bouvet@

et douches des hôpi-

bch.ap-hop-paris.fr

taux, des hôtels et des campings, bains à remous, fontaines.

De nombreuses études ont été réalisées pour identifier les facteurs de risque de contamination des installations à risque, et des actions de prévention systématique visant ces installations sont maintenant organisées avec une certaine efficacité. Le risque nosocomial a été le premier visé; des mesures préventives fondées sur la surveillance des réseaux de distribution d'eau chaude dans les hôpitaux ont été engagées depuis les années 80 avec une efficacité certaine, puisque le nombre de cas en milieu hospitalier a significativement diminué en France $[2,3]$.

\section{Caractéristiques de Legionella pneumophila}

Legionella pneumophila est un bacille intracellulaire à Gram négatif, cultivable sur milieu spécifique $B C Y \varepsilon a$ (buffered charcoal yeast extract). Le genre comprend 49 espèces et plus de 64 sérogroupes. Legionella pneumophila sérogroupe 1 (LPl) est l'organisme le plus fréquemment retrouvé en pathologie humaine (environ $90 \%$ des cas), suivi par le sérogroupe 6 . À ce jour, outre Legionella pneumophila, 19 espèces ont été retrouvées pathogènes pour l'homme.

Il s'agit d'une bactérie d'origine hydrotellurique, qui colonise de façon ubiquitaire les eaux douces naturelle (lacs, rivières) et les sols humides, ainsi que de nombreux milieux artificiels tels que les eaux chaudes sanitaires et l'eau des tours aéroréfrigérantes.

Article reçu le 15 mars 2006, accepté le 5 mai 2006. 
Certaines conditions favorisent sa multiplication et sa survie : une température de l'eau située entre $25^{\circ} \mathrm{C}$ et $42^{\circ} \mathrm{C}$, sa stagnation, la présence de biofilm et de dépôts de tartre ou de corrosion [4]. Ainsi, de nombreuses études montrent que les biofilms présents dans les installations représentent un réservoir important de légionelles, qui peuvent ensuite être détachées et entraînées avec le flux. De plus, cette présence de biofilm, en protégeant les légionelles de l'action des traitements biocides, pourrait expliquer l'échec des procédures de désinfection engagées [4]. Les légionelles peuvent, par ailleurs, coloniser d'autres micro-organismes, notamment les amibes ou d'autres protozoaires ciliés, dans lesquels elles peuvent survivre et résister aux procédés de désinfection, voire se développer et contaminer le milieu extérieur après lyse de leur hôte $[5,6]$; cette interaction avec les amibes pourrait augmenter leur pathogénicité. La concentration en Legionella pneumophila au niveau du réservoir est certainement un élément important dans l'intensité du risque d'infection. Cependant, la dose infectante pour l'homme n'est pas clairement définie [7]. Le risque semble plus important au-dessus du seuil de 1000 UFC/I (unité formant colonie/litre), mais ce niveau doit être modulé en fonction du terrain de l'hôte. Des études ont montré qu'au sein d'un même point d'émission d'eau, la concentration de légionelles peut être très fluctuante. Des légionelles sont fréquemment isolées dans l'eau chaude domestique, avec un niveau moyen de contamination dans les prélèvements positifs dépassant légèrement 1000 UFC/I. C'est ainsi qu'une étude italienne récente retrouve une contamination de l'eau chaude domestique par des légionelles dans $22,6 \%$ des prélèvements [8], tandis que des études antérieures finlandaises et allemandes retrouvaient un taux de positivité de $30 \%$ et $26 \%$, respectivement. Toutes ces études confirment que Legionella pneumophila est de loin l'espèce bactérienne la plus abondante dans l'eau potable ou environnementale.

La contamination des points d'eau peut intervenir toute l'année. La concentration en certains sels minéraux tels que le cuivre ou le zinc pourrait jouer un rôle dans leur développement $[8,9]$. Legionella pneumophila est sensible à la chaleur: au-delà de $60^{\circ} \mathrm{C}$, les légionelles sont tuées. D'autres procédés physiques et chimiques tels que la chloration peuvent être utilisés pour réduire la contamination des réseaux, même si Legionella pneumophila y est relativement tolérante. Si la chloration continue permet de réduire le degré de contamination de l'eau de distribution, son utilisation est toutefois discutée, car elle pose d'autres problèmes, notamment ceux d'une corrosion des canalisations et de la production de produits de dégradation potentiellement cancérigènes [10].

\section{Sources potentielles de contamination}

À partir du milieu naturel, la bactérie colonise des sites hydriques artificiels lorsque les conditions de son développement sont réunies. Sa prolifération est ainsi favorisée par les conditions présentes dans différentes installations à risque telles que les réseaux d'eau chaude, les circuits des tours aéroréfrigérantes, les bains à bulle et les humidificateurs.

\section{Circuits de distribution d'eau chaude sanitaire}

II s'agit des circuits alimentant les douches ou les autres points d'arrivée d'eau. Différents facteurs sont associés au risque de dévelop- pement des légionelles: une distance entre le point de chauffage et le point d'usage supérieure à 10 mètres, l'existence d'un chauffage central et une ancienneté du système de plus de 10 ans sont associées à un risque plus élevé de contamination, tandis que des concentrations de cuivre $>50 \mathrm{mg} / \mathrm{l}$ et de zinc $<100 \mathrm{mg} / \mathrm{l}$ sont prédictives d'absence de colonisation du système.

\section{Systèmes de refroidissement}

\section{et tours aéroréfrigérantes humides}

Les circuits chauds industriels ou les groupes frigorifiques utilisés en climatisation, en froid industriel ou commercial, sont des milieux favorables au développement des légionelles, en raison de la température de l'eau et du contact air/eau dans ces installations. Les tours aéroréfrigérantes situées à l'extérieur des bâtiments sont des systèmes de refroidissement de ces circuits chauds: elles évacuent la chaleur vers l'extérieur en pulvérisant l'eau en fines gouttelettes dans un flux d'air circulant à contre courant, grâce à un ventilateur. Cette circulation forcée de l'air provoque l'entraînement d'un aérosol de gouttelettes présent dans le panache. En région parisienne, entre 1995 et 1999, une étude effectuée par le laboratoire d'hygiène de la ville de Paris a mis en évidence que $75 \%$ des équipements contrôlés présentaient des concentrations égales ou supérieures à $100 \mathrm{UFC/I}$, et $55 \%$ des concentrations supérieures à 1000 UFC/I.

Une épidémie de légionellose humaine de grande importance, survenue dans le Pas-de-Calais en 2003-2004 ( 86 cas entre le 5 novembre 2003 et le 22 janvier 2004), a été associée à la diffusion des bactéries sur des distances supérieures à $10 \mathrm{~km}$, ce qui a été vérifié par des simulations de dispersion des gouttelettes émises par la tour aéroréfrigérante. Des sources puissantes telles que des tours aéroréfrigérantes industrielles peuvent donc, dans des conditions météorologiques particulières, émettre des particules contaminées à une distance très grande, ce qui permet d'expliquer aussi bien les épidémies que certains cas sporadiques $[1,11]$.

\section{Bassins utilisés pour la détente}

Des installations de balnéothérapie ou de thermalisme dans lesquelles l'eau chaude est dispersée par des systèmes d'injection d'air ou d'aérosols ont été à l'origine de cas groupés. Deux épidémies ont ainsi été occasionnées en 1999, par l'exposition à l'eau d'un bain à remous présenté lors de grandes expositions: I'une aux Pays-Bas, en mars 1999 (124 cas), l'autre en Belgique, en décembre 1999 (93 cas) [12].

D'autres cas ont été associés à des fontaines décoratives, des équipements de thérapie respiratoire par aérosol 
ou des machines à glace [1]. En revanche, les systèmes de traitement d'air de type batterie froide sont, par leur conception, peu susceptibles d'être colonisés par des légionelles et de contaminer la population.

Parmi toutes les sources de contamination, les circuits d'eau chaude et les tours aéroréfrigérantes sont les plus fréquemment impliquées dans la survenue de cas humains. Néanmoins, plus de la moitié des cas survient sans facteur d'exposition identifiable, soulevant la question d'une possible contamination des réseaux d'eau chaude domestique ou de sources environnementales intermittentes non repérées [13, 14].

\section{Modes de transmission}

La légionellose est transmise par inhalation de micro-gouttelettes d'eau, de diamètre inférieur à 5 microns. La possibilité de contamination par micro-aspiration est discutée; elle pourrait intervenir par «fausse route » avec l'eau de boisson (personnes âgées), ou chez des sujets porteurs d 'une sonde œsogastrique, souffrant de troubles de la déglutition [15] ou opérés de la sphère ORL. Le risque de contamination par l'ingestion d'eau contaminée est elle aussi discutée: aucun argument formel ne confirme cette hypothèse. Enfin, il n'existe pas de transmission de personne à personne.

\section{Épidémiologie}

\section{Systèmes de surveillance}

La légionellose est surveillée en France par deux systèmes $[2,3]$ : la déclaration obligatoire (Do) des cas par les cliniciens ou les laboratoires qui font le diagnostic, et le centre national de référence (CNR) qui reçoit les souches provenant des laboratoires de microbiologie (Figure l).

La déclaration obligatoire s'effectue en deux temps: dès que le diagnostic est posé, le signalement systématique du cas par les cliniciens ou les biologistes à la DDASS permet d'intervenir, dans des délais réduits, sur la source présumée. Dans un second temps, la notification

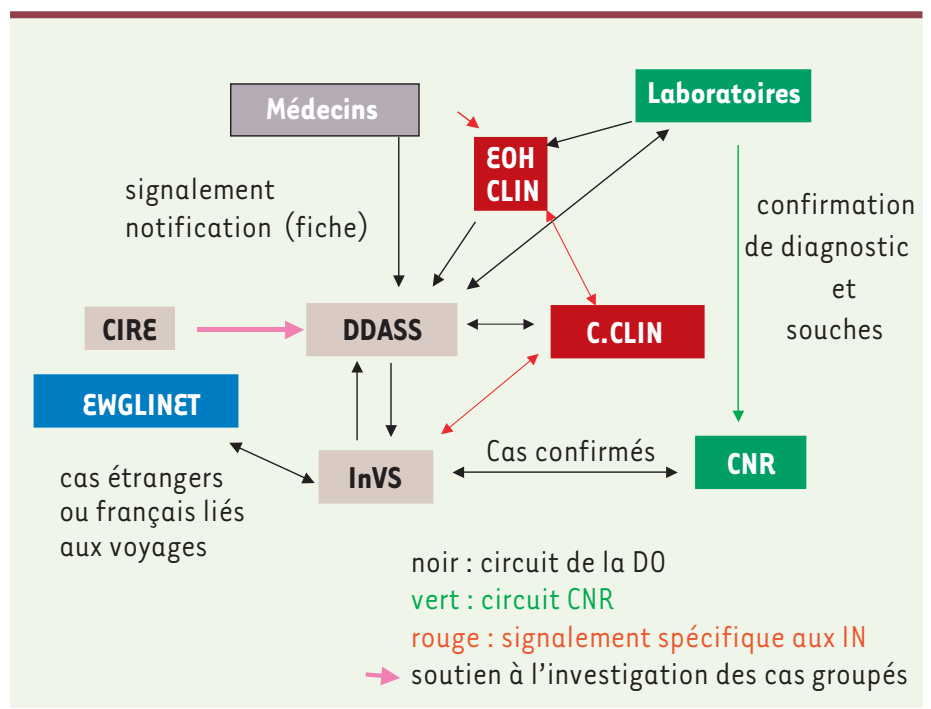

Figure 1. Circuits de déclaration des légionelloses (Institut de Veille Sanitaire, InVS). est réalisée par le clinicien grâce à l'envoi d'une fiche anonymisée à la DDASS. Le CNR réalise des diagnostics de première intention et reçoit des prélèvements pour confirmation. II notifie systématiquement tous ces cas à l'Institut de veille sanitaire (InVS), permettant ainsi de signaler ceux qui n'auraient pas été déclarés. Toutes les souches isolées par les laboratoires doivent être envoyées au CNR afin que soit étudié leur profil génomique. Les souches provenant des patients et celles de la (ou des) source(s) potentielle(s) peuvent donc être comparées, ce qui aide à déterminer l'origine de la contamination. La banque de données informatisée rassemblant tous les profils de macrorestriction, qui existe depuis 1998, contient actuellement plus de 4000 souches, dont 1000 sont cliniques [16-18].

Au niveau européen, la France participe au réseau EWGLI (European working group on Legionella infections). Ce réseau, auquel participent 36 pays, signale aux autorités sanitaires du pays concerné tout cas survenu chez une personne ayant voyagé dans les 10 jours précédant le début de la maladie. Les cas à déclarer comme cas confirmés sont les pneumopathies associées à un critère biologique d'infection: isolement de Legionella dans un prélèvement clinique, ascension du titre des anticorps (avec un titre supérieur ou égal à 1/128), présence d'antigène soluble dans les urines ou immunofluorescence directe positive dans les sécrétions respiratoires. Les cas probables sont définis par un titre unique d'anticorps supérieur ou égal à 256 (1/256).

\section{Données}

Le nombre des cas déclarés en France (Figure 2) était de 1044 en 2003 et de 1202 en 2004, soit une incidence de 2 cas pour 100000 habitants (1/100000 en Europe en 2003). La mortalité était de $14 \%$ en 2003 comme en 2004 (138 décès pour 1013 cas avec évolution connue) [2, 3]. La légionellose est responsable de $0,5 \%$ à $5 \%$ des pneumopathies communautaires nécessitant une hospitalisation. Les légionelloses d'origine nosocomiale représentaient quant à elles $9 \%$ des cas déclarés en 2003 , et $6 \%$ en 2004 [19]. En 2003 , les patients constituant les cas déclarés avaient un âge médian de 62 ans; le sex ratio était de 2,6. L'incidence la plus élevée était retrouvée chez les hommes âgés de plus de 80 ans. Un ou plusieurs facteurs favorisants ont été retrouvés dans $69 \%$ des cas en 2003 : cancer ou hémopathie (10\%), corticothérapie prolongée ou récente et à forte dose ou immunosuppression ( $9 \%$ ), diabète (11\%) et tabagisme (42\%) (Tableau I).

Depuis 2000, en France, une exposition à risque dans les 10 jours précédant le début des symptômes est rapportée pour la moitié des cas. Les situations 
ayant constitué un risque possible d'exposition sont un séjour dans un hôpital ou une clinique dans $10 \%$ des cas, un séjour dans un camping ou un hôtel dans près de $15 \%$ des cas et la notion de voyage dans $17 \%$ des cas. Les cas groupés épidémiques impliquant plus de 10 cas représentaient 121 cas en 2003 , et 53 en 2002 .

Sur le plan de la distribution temporelle, on retrouve habituellement une nette augmentation des cas pendant la période estivale [19].

\section{Clinique}

\section{Diagnostic}

Les légionelloses se manifestent sous deux formes cliniques distinctes. La fièvre de Pontiac, caractérisée par un syndrome pseudogrippal bénin sans pneumonie, n'est pas soumise à la déclaration obligatoire; sa durée d'incubation varie de 5 heures à 4 jours, et sa guérison est spontanée en 2 à 5 jours. La pneumopathie à légionelle, ou maladie des légionnaires, est quant à elle surveillée par la déclaration obligatoire; elle se manifeste essentiellement par une symptomatologie respiratoire (toux sèche, dyspnée, douleur thoracique, fièvre élevée) d'évolution habituellement progressive et sévère et, souvent, par des signes extrarespiratoires devant attirer l'attention

\begin{tabular}{lcc}
\hline Facteurs prédisposants & N & $\%$ \\
Cancer/hémopathie & 101 & 10 \\
Corticothérapie/immunosuppression & 96 & 9 \\
Diabète & 117 & 11 \\
Tabagisme & 439 & 42 \\
Autres & 225 & 22 \\
$\geqslant \mathbf{1}$ facteur & $\mathbf{7 2 3}$ & $\mathbf{7 1}$ \\
\hline
\end{tabular}

Tableau I. Facteurs de prédisposition à la légionellose (non mutuellement exclusif) (données de déclaration obligatoire, Institut de Veille Sanitaire, France, 2003).

Nombre de cas

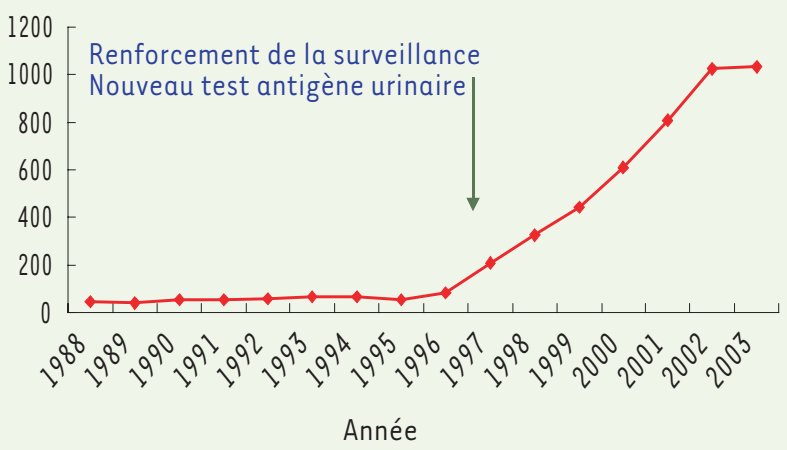

Figure 2. Évolution du nombre de cas de légionellose déclarés en France (19882003) (Données de déclaration obligatoire, Institut de Veille Sanitaire). (diarrhée, myalgies, céphalées intenses, confusion, dissociation du pouls et de la température). Plusieurs éléments biologiques évocateurs sont souvent retrouvés, tels qu'une hyponatrémie, une cytolyse hépatique ou une absence d'hyperleucocytose à polynucléaires. Les images radiologiques pulmonaires sont variables, avec souvent une atteinte bilatérale, non systématisée, caractérisée par une atteinte interstitielle ou alvéolo-interstitielle.

Le diagnostic, qui doit être évoqué devant toute pneumopathie, peut être effectué par plusieurs techniques [20]. La culture des prélèvements respiratoires reste la méthode de référence. Elle peut être réalisée à partir des expectorations ou de tout autre prélèvement bronchopulmonaire (aspiration trachéale, lavage bronchoalvéolaire, brosse...). La culture, lente, s'effectue sur un milieu spécifique tel que le BCyE; elle seule permet de caractériser la souche.

La recherche d'antigènes solubles dans les urines est la meilleure technique pour obtenir un diagnostic rapide (en 15 minutes par immunochromatographie sur membrane, en 4 heures par Elisa). Elle devient positive précocement, dans les 2 à 3 jours après l'apparition des symptômes, et reste positive malgré un traitement antibiotique adapté pendant plusieurs semaines. Cependant, les tests utilisés actuellement ne détectent que Legionella pneumophila de sérogroupe 1 (qui est tout de même responsable de près de $90 \%$ des cas en France). Sa valeur prédictive positive est de l'ordre de $86 \%$, et sa valeur prédictive négative de $95 \%$.

L'immunofluorescence directe des prélèvements pulmonaires permet un diagnostic rapide, mais suppose des manœuvres invasives et reste peu sensible et peu spécifique. Quant à la sérologie, elle permet un diagnostic tardif, voire rétrospectif, et son résultat a rarement un impact sur le traitement dans la mesure où elle ne devient positive que tardivement (entre 10 jours et 4 semaines). Seule la mise en évidence d'une augmentation par 4 du titre des anticorps par immunofluorescence indirecte ou Elisa est significative. Elle permet souvent le diagnostic rétrospectif des légionelloses dues aux sérogroupes non-1. Elle possède surtout une valeur épidémiologique.

La réalisation d'une PCR sur prélèvement respiratoire, sérum ou urine permet un diagnostic rapide. La sensibilité de cette méthode de diagnostic serait comprise entre $80 \%$ et $100 \%$ pour les prélèvements respiratoires, et sa spécificité supérieure à $90 \%$ pour tous les types de prélèvements. Elle n'est encore ni normalisée, ni incluse dans les critères diagnostiques de légionellose dans l'Union européenne. 


\section{Traitement}

Le traitement de la légionellose [21] repose sur l'antibiothérapie, qui doit être administrée rapidement: le délai de mise en route d'une antibiothérapie adaptée est en effet un facteur important de la guérison.

Les antibiotiques actifs sur les légionelles sont les macrolides, qui constituent toujours le traitement de référence, les fluoroquinolones et la rifampicine, qui doit toujours être utilisée en association. Les $\beta$-lactamines ne doivent pas être utilisées, car elles sont constamment inactives. On traite habituellement les formes de gravité légère à modérée par un macrolide ou une fluroquinolone, en monothérapie, pendant une durée de 14 à 21 jours. Dans les formes graves, il est recommandé d'associer deux antibiotiques: fluoroquinolone + macrolides ou rifampicine ou macrolide + rifampicine pour une durée plus longue, jusqu'à 30 jours.

II n'existe pas d'argument scientifique pour recommander une prophylaxie par antibiotique en situation de possible exposition, en particulier chez des patients à risque tels que des sujets immunodéprimés. Toutefois, on peut proposer d'utiliser un macrolide chez les sujets les plus à risque [22].

\section{Prévention}

La première mesure préventive est d'intervenir sur les sources potentielles. Elle consiste à limiter ou supprimer la contamination de l'eau par les légionelles des installations à risque de transmission. Dans différents cas, la suppression de certains types d'installation particulièrement à risque doit être envisagée. Par ailleurs, les patients ayant des facteurs prédisposants à l'infection par les légionelles doivent, quand cela est possible, éviter de fréquenter les installations comportant un risque élevé de contamination.

Les premières mesures réglementaires préconisées dans les années 90 concernaient les établissements de santé, et avaient pour objectif d'agir sur les systèmes de distribution d'eau chaude à trois niveaux: éviter la stagnation en assurant une bonne circulation de l'eau ; lutter contre l'entartrage et la corrosion; maintenir l'eau à une température élevée depuis la production jusqu'au plus près des points d'usage [23]. II est admis que la mise en œuvre de ces actions permet de limiter, voire de supprimer la nécessité de réaliser des interventions curatives ponctuelles sur le réseau, tels que des chocs chlorés ou thermiques [22].

D'autres textes et procédures ont été élaborés depuis pour limiter et gérer les risques liés aux autres types d'installation : les tours aéroréfrigérantes humides, notamment, sont visées par des dispositions réglementaires de 2004 [10], qui prévoient la mise en place d'un plan de nettoyage et de désinfection, afin que la concentration en légionelles dans l'eau du circuit de refroidissement reste inférieure à $1000 \mathrm{UFC/I}$, et la mise en place d'un plan de surveillance, au minimum trimestriel, destiné à s'assurer de l'efficacité du nettoyage et de la désinfection.

\section{Conclusions}

La bactérie responsable de légionellose, maintenant bien connue, constitue un exemple d'hôte tout à fait habituel de l'environnement hydrique, pouvant être transmis à l'homme par inhalation d'aérosols contaminés à partir du réservoir. Différents types d'installations industrielles ou balnéaires favorisant la production d'aérosols peuvent être à l'origine de contaminations humaines parfois très nombreuses, avec risque d'épidémies dont il est encore souvent difficile de retrouver la source.

Depuis les années 90, de nombreux textes réglementaires ont été publiés, destinés à imposer des mesures de surveillance et de prévention de la contamination par Legionella pneumophila dans plusieurs types d'établissements, notamment les réseaux d'eau chaude hospitaliers et les tours aéroréfrigérantes. Des effets positifs notables de l'adoption de ces réglementations ont été enregistrés notamment en milieu hospitalier, avec la nette réduction des cas nosocomiaux observée depuis 3 ans en France. $\diamond$

\section{SUMMARY}

\section{Legionellosis}

Although one does not find the origin of the contamination in the human half of the cases of legionellosis, one knows that this disease is the consequence of the almost obligatory contamination of the networks of installations of hot water by Legionella pneumophila, and the inhalation by the man of infected droplets. Pathology generally consists of a relatively serious pneumopathy. The control of the level of contamination of the various producing hydrous installations of aerosols is imperative to avoid the serious medical consequences, which cannot be prevented by an action on the human target. The majority of the currently identified tanks are the air and cool towers and the distribution networks of hot water. The taking into account of this risk in the hospitals or thermal led to the implementation of many measurements of disinfection and control, which start to show a certain effectiveness on which has occurred of new cases in these establishments, today in clear reduction. $\diamond$

\section{RÉFÉRENCES}

1. Desenclos JC. La legionellose: de Philadelphie au Pas de Calais! BEH 2004 ; 36-37: 173.

2. Campèse $C$, Jarraud $S$, Bitar $D$, et al. Les légionelloses survenues en France en 2004. BEH 2005; 26 : 129-32.

3. Campèse $C$, Jarraud $S$, Decludt $B$, et al. Les légionelloses déclarées en France en 2003. BEH 2004 ; 36-37 : 174-6.

4. Murga R, Forster TS, Brown $\varepsilon$, et al. Role of biofilms in the survival of Legionella pneumophila in a model potable water system. Microbiology 2001 ; $147: 3121-6$

5. Abu Kwaik Y, Gao Ly, Stone BJ, et al. Invasion of protozoa by Legionella pneumophila and its role in bacterial ecology and pathogenesis. Appl Environ Microbiol $1998 ; 64: 3127-33$.

6. Kilvington S, Price J. Survival of Legionella pneumophila within cysts of Acanthamobea polyphaga following chlorine exposure. J Appl Bacteriol 1990 ; $68: 519-25$.

7. Atlas RM. Legionella : from environmental habitats to disease pathology, detection and control. Environ Microbiol 1999; 1 : 283-93.

8. Borella P, Montagna MT, Romano-SpicaV, et al. Legionella infection risk from domestic hot water. Emerg Infect Dis 2004 ; 10 : 457-64.

9. CDC Legionellosis. www.cdc.gov 
10. Conseil supérieur d'hygiène publique de France (CSHPF). Le risque lié aux légionelles. Guide d'investigation et d'aide à la gestion. ler juillet 2005. Ministère de la santé. www.sante.gouv.fr

11. Rouil L, Gardenas G, Marcel F. Évaluation de la dispersion atmosphérique d'aérosols potentiellement contaminés lors de l'épidémie de légionellose de la région de Lens. BEH $2004 ; 36-37: 182-4$.

12. Den Boer JW, Yzerman \& PF, Shellekens J, et al. A large outbreak of Legionnaires' disease at a lower show, the Netherlands, 1999. Emerging Infect Dis $2002 ; 8: 37-43$.

13. Mudder RR, Yu VL, Fang GD. Community acquired Legionnaires' disease. Semin Respir Infect $1989 ; 4: 32-9$.

14. Che D, Campese C, Decludt B, Desenclos JC. Sporadic cases of community acquired legionnaires' disease : an ecological study to identify new sources of contamination. J Epidemiol Community Health $2003 ; 57$ : 466-9.

15. Neill M, Gorman VW, Gibert C, et al. Nosocomial legionellosis, Paris, France. Evidence for transmission by potable water. Am J Med $1985 ; 73: 581-8$.

16. Fry NK, Alexiou-Daniel S, Bangsborg JM, et al. A multicenter evaluation of genotypic methods for the epidemiologic typing of Legionella pneumophila serogroup 1 : results of a panEuropean study. Clin Microbiol Infect 1999; 5 : 462-77.

17. Gaia V, Fry NK, Harrison TG, Peduzzi R. Sequence-based typing of Legionella pneumophila serogroup 1 offers the potential for true portability in legionellosis outbreak investigation. J Clin Microbiol 2003 ; 41 : 2932-9.
18. Pruckler JM, Mermel LA, Benson RF, et al. Comparison of Legionella pneumophila isolates by arbitrarily primed PCR and pulsed-field gel electrophoresis : analysis from seven epidemic investigations. J Clin Microbiol $1995 ; 33: 2872-5$.

19. Sabria M, Yu VL. Hospital-acquired legionellosis : solutions for a preventable infection. Lancet Infect Dis 2002 ; 2 : 368-73.

20. Murdoch DR. Diagnosis of Legionella infection. Clin Infect Dis 2003 $36: 64-9$.

21. Edelstein PH. Antimicrobial chemotherapy for Legionnaires' disease : a review. Clin Infect Dis $1995 ; 21: 5265-76$.

22. CSHPF. Avis du 27 mai 2005 relatif à la place de l'antibioprophylaxie dans la prévention des légionelloses nosocomiales.

23. DGS/DHOS. Circulaire $n^{\circ} 2002 / 243$ du 22 avril 2002 relative à la prévention du risque lié aux légionelles dans les établissements de santé.

\section{LA RAPIDITÉ ET LA SIMPLICITÉ AVANT TOUT !}

\section{Promega vous propose ses technologies}

\section{pour détecter la VIABILITÉ CELLULAIRE \& L'APOPTOSE !}

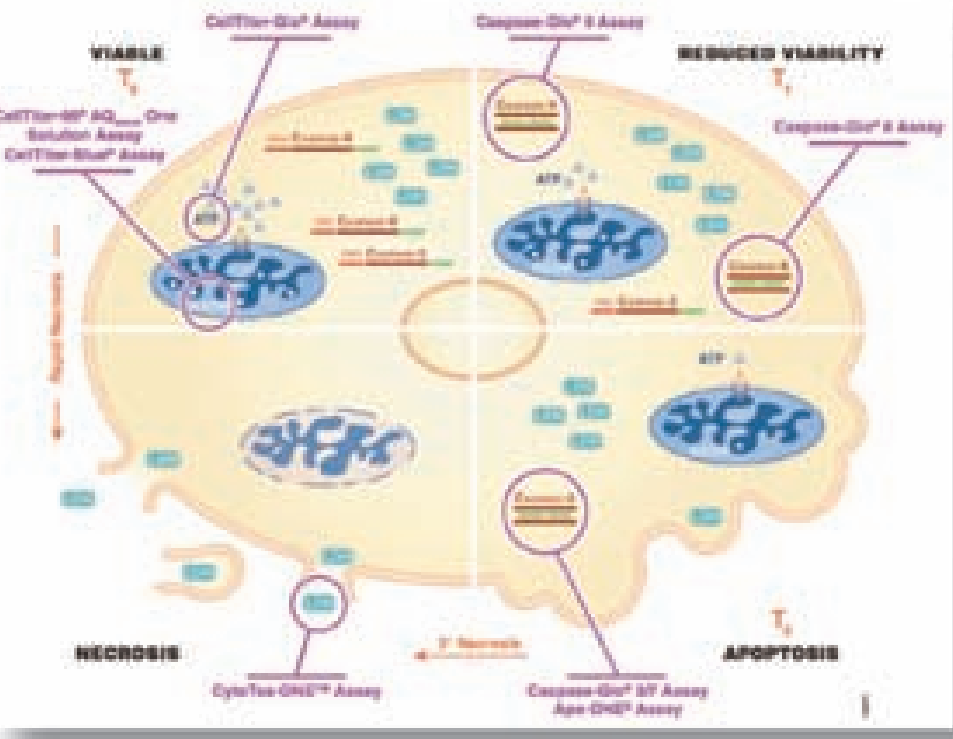

Une seule êtape

directement sur cellules en culture

- Ultra sensibles

(à partir de quelques dizaines de cellules)

Disponibles pour tous types d'appareillages (fluorimètre, luminomètre et spectrophotomètre)

\section{Pour plus d'information consultez le site : www.promega.com/paguide/ ou contactez le 0800488000 .}

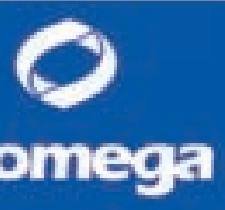

